\title{
Caracterización de la Citología Exfoliativa Vaginal en Alpacas (Vicugna pacos)
}

\author{
Characterization of Vaginal Exfoliative Cytology in Alpacas (Vicugna pacos) \\ Joel Pacheco C. ${ }^{1,2}$
}

\section{Resumen}

Se realizó una caracterización de las formas celulares encontradas en frotices vaginales de alpacas vacías, así como la caracterización del índice de maduración en diferentes estadios fisiológicos (hembras vacías, preñadas, posparto temprano, pos-cópula) y la descripción del tejido vaginal de hembras vacías y preñadas para determinar diferencias estructurales. Las diferencias del índice de maduración entre alpacas vacías y preñadas podrían ser útiles como método de diagnóstico de gestación.

Palabras clave: citología vaginal exfoliativa; frotices vaginales; índice de maduración; alpaca

\section{Abstract}

The characterization of the cellular forms found in vaginal smears of non-pregnant alpacas was carried out, as well as the characterization of the maturation index in various physiological stages (non-pregnant, pregnant, early post-partum, post-copula) and the description of the vaginal tissue of non-pregnant and pregnant females to determine structural differences. Differences in the maturation index between non-pregnant and pregnant alpacas could be useful as a diagnostic method of gestation.

Key words: exfoliative vaginal cytology; vaginal smears; maturation index; alpaca

\footnotetext{
${ }^{1}$ Estación Experimental Maranganí, Centro de Investigación IVITA, Facultad de Medicina Veterinaria, Universidad Nacional Mayor de San Marcos, Cusco, Perú

${ }^{2}$ Email: jpachecoc@unmsm.edu.pe
}

Recibido: 31 de enero de 2017

Aceptado para publicación: 5 de julio de 2017 


\section{INTRODUCCIÓN}

La vagina es un órgano muscular compuesto por tres capas: mucosa, muscular y adventicia; revestida por epitelio estratificado plano no queratinizado. Este epitelio sufre variaciones debido a cambios hormonales que ocasionan la muerte y descamación de las células de las capas superficiales (Jones y Edgren, 1973; Dellman y Brown 1980; Feldman y Nelson, 2007). Cambios en la estructura histológica de la vagina, incluyendo el aumento de la irrigación sanguínea, son debidos a la edad y el número de partos del animal (Rodríguez et al., 2004).

La citología exfoliativa vaginal es una técnica simple, que se utiliza con mucha eficacia para determinar el estadio del ciclo estral en perras y gatas, dado que los niveles cambiantes de estrógenos en sangre producen variaciones morfológicas en el epitelio vaginal (Thrall y Olson, 2003). Asimismo, la actividad mitótica del estrato basal del epitelio de la vagina en la oveja se incrementa a medida que se acerca el momento de la ovulación, mientras que se encuentran pocas células superficiales descamadas en el diestro (Hawkins y Darlow, 1934; Porto et al., 2007).

Las células encontradas en frotis vaginales han sido clasificadas como células parabasales (redondas con poco citoplasma), células intermedias pequeñas y grandes (células mas grandes, núcleo grande, ligeramente ovaladas) y células superficiales (anucleadas y con núcleo picnótico, citoplasma irregular) (Piñeiro et al., 1995; Thrall y Olson, 2003). Por otro lado, el índice de maduración es el porcentaje de células parabasales, intermedias y superficiales encontradas en frotis vaginales, el cual se puede relacionar a los estadios del ciclo reproductivo del animal en estudio (Mills et. al. 1979).

Las dimensiones de los diferentes tipos de células encontradas en frotis vaginales de alpacas corresponden a 18-25, 29-43 y 28-78 $\mu \mathrm{m}$ de diámetro para células parabasales, intermedias y superficiales respectivamente (Pedrana et al., 2008).

En el canino se observa un $80 \%$ de células superficiales en la fase de estro y de $20 \%$ en el diestro, mientras que en el anestro solo se observan células parabasales e intermedias pequeñas (Stornelli et al., 2006), en tanto que en la gata se observa $88 \%$ de células superficiales en el estro, y de $87 \%$ de células intermedias y $3 \%$ de células superficiales en el anestro (Mills et al., 1979). En especies como el pecarí de collar (Tayassu tajacu) se indica que $60 \%$ o más de células superficiales es un indicador del estro (Mayor et al., 2004).

El diagnóstico de gestación mediante citología exfoliativa está reportado en monos del género Aotus (Ríos et al., 1999) y Callitrix (Mitchell y Jones, 1975), así como en la chinchilla (Chinchilla lanigera) y el hurón (Mustela spp), donde las diferencias entre los índices de maduración de hembras vacías y preñadas son evidentes (Williams et al., 1992; Bekyürek et al., 2002).

En los camélidos sudamericanos, debido a la singularidad de su fisiología ovárica, esta técnica podría ser de ayuda en el manejo reproductivo, así como una alternativa para el diagnóstico de gestación, en especial en especies silvestres como vicuñas y guanacos, dado su carácter de técnica no invasiva. Los objetivos del presente estudio fueron determinar las características morfológicas de las células obtenidas mediante frotis vaginales, determinar el índice de maduración en varios estados fisiológicos (vacías, preñadas, posparto temprano y poscópula) y determinar las diferencias estructurales del epitelio vaginal entre hembras vacías y preñadas.

\section{Materiales y MéTodos}

Experimento I: Descripción citológica de células vaginales 
Se obtuvieron frotis vaginales de 10 alpacas adultas durante la estación reproductiva. Para esto, un hisopo de $10 \mathrm{~cm}$ fue introducido $7 \mathrm{~cm}$ dentro de la vagina, donde se realizó movimientos rotatorios contra la pared vaginal. Luego, se hizo rodar el hisopo en una lámina portaobjetos, se fijó con alcohol etílico de $90^{\circ}$ y se dejó secar. Los frotis fueron coloreados con la tinción Wright por $5 \mathrm{~min}$, se adicionó agua tamponada por 3 min, se lavó con agua corriente, y finalmente se dejó secar. Los frotis fueron observados en microscopio óptico a diferentes aumentos. La medición de las células se realizó utilizando un microscopio de proyección a 400 aumentos.

Experimento II: Citología exfoliativa vaginal según el estado reproductivo

Se obtuvieron frotis vaginales de 10 alpacas vacías (5 aceptan la cópula y 5 rechazan la copula), 10 alpacas preñadas y 12 alpacas en posparto temprano (2-5 días posparto). Todos los animales eran adultos y las muestras fueron recolectadas durante la estación reproductiva.

Además, se obtuvieron frotis vaginales de tres alpacas poscópula (días 3, 6, 9 y 12) $\mathrm{y}$ los resultados fueron comparados con hallazgos en la observación de los órganos reproductivos como presencia de cuerpo lúteo y presencia / ausencia de embrión. La tinción de los frotis se hizo siguiendo el procedimiento indicado en el Experimento I.

Experimento III: Modificaciones estructurales en vagina de alpacas vacías y preñadas

Dos alpacas vacías y dos preñadas fueron sacrificadas y se tomaron muestras vaginales para estudio histológico. Los tejidos fueron fijados con paraformaldehido al $4 \%$ diluido en PBS a $0.1 \mathrm{M}$ y pH 7.2, fueron sometidos a procedimientos histológicos de rutina. Los cortes fueron de $7 \mu \mathrm{m}$ de grosor, coloreados con hematoxilina y eosina, y evaluados en microscopio óptico.
Las alpacas utilizadas en los experimentos II y III fueron sacrificadas en el matadero municipal del Distrito de Nuñoa, Puno. Los análisis estadísticos para los experimentos II y III se realizaron mediante la prueba de Chicuadrado.

\section{Resultados y Discusión}

\section{Experimento I}

Se pudo observar la existencia de formas celulares similares a las reportadas en especies como caninos y felinos (Piñeiro et al., 1995; Thrall y Olson, 2003), descritas como parabasales, intermedias (pequeñas y grandes) y superficiales.

En la Figura 1 se muestran los tipos de células encontradas en los frotis vaginales. Las células parabasales eran de forma redondeada, con un núcleo de gran tamaño y escaso citoplasma, formando generalmente columnas celulares agrupadas. Las células intermedias pequeñas se observaron como células redondeadas, de mayor tamaño que las células parabasales y con mayor cantidad de citoplasma, pero con el núcleo de similar tamaño a las parabasales. Las células intermedias grandes presentaban forma ovalada y la presencia de mayor cantidad de citoplasma era notorio al poseer un núcleo más pequeño. En algunas células se observaba un inicio de picnosis, pudiéndose confundirse con células superficiales de núcleo picnótico.

Las células superficiales se presentan como células grandes de forma irregular, angulosas y, en algunos casos, se muestran plegadas. En el caso de células superficiales con núcleo picnótico, el núcleo se encuentra fragmentado, débilmente teñido y en franca degeneración (Figura 1F). También se observan células anucleadas, cuya tinción es menos intensa por estar altamente queratinizadas (Figura 1E). Se pueden observar leucocitos, especialmente neutrófilos (Figura 1G), pero muy pocos eritrocitos. 


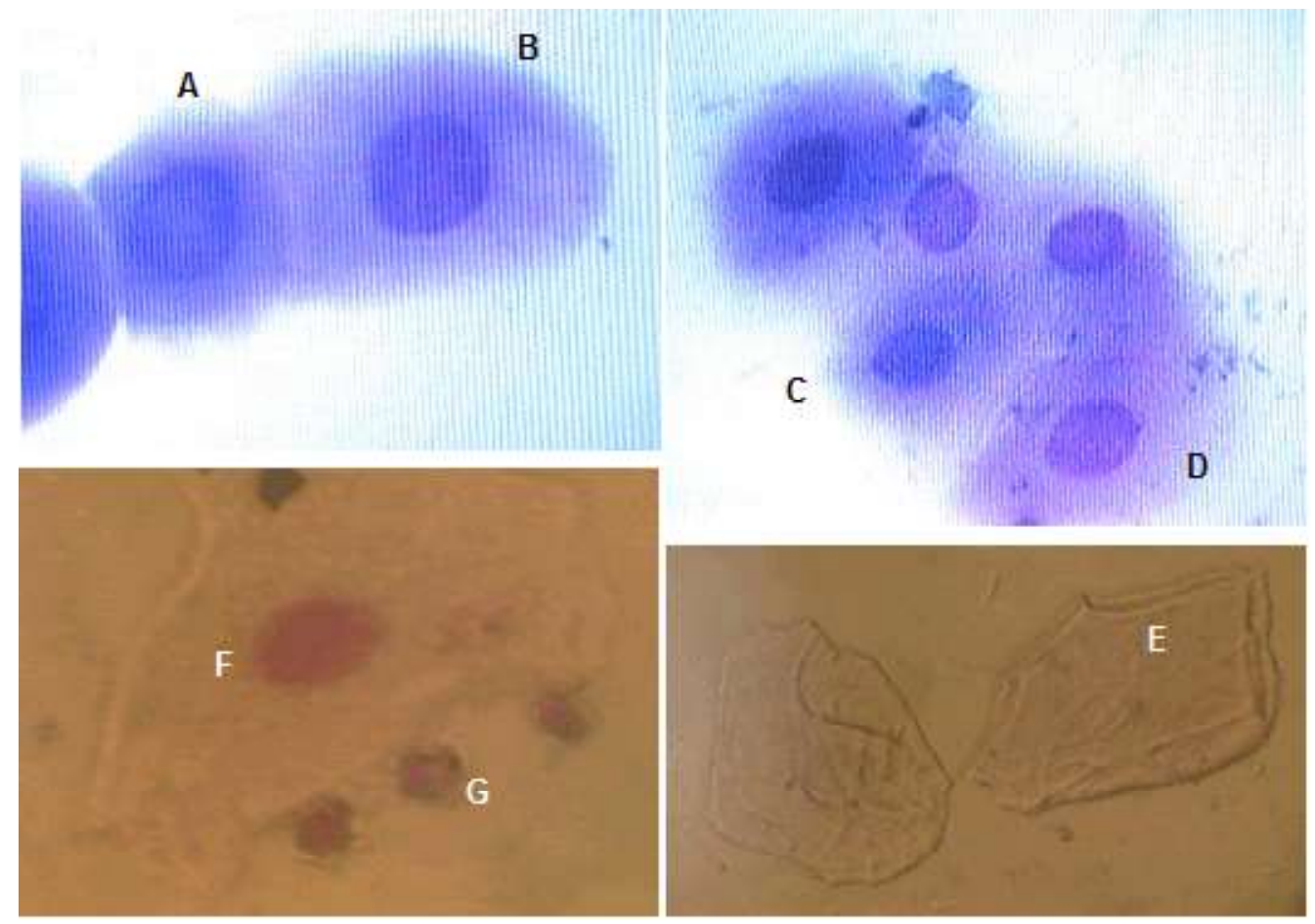

Figura 1. Células parabasales, intermedias y superficiales en frotices vaginales de alpaca. A: célula parabasal; B y C: células intermedias pequeñas; D: célula intermedia grande; E: célula superficial anucleada; F: célula superficial con núcleo picnótico; G: neutrófilos

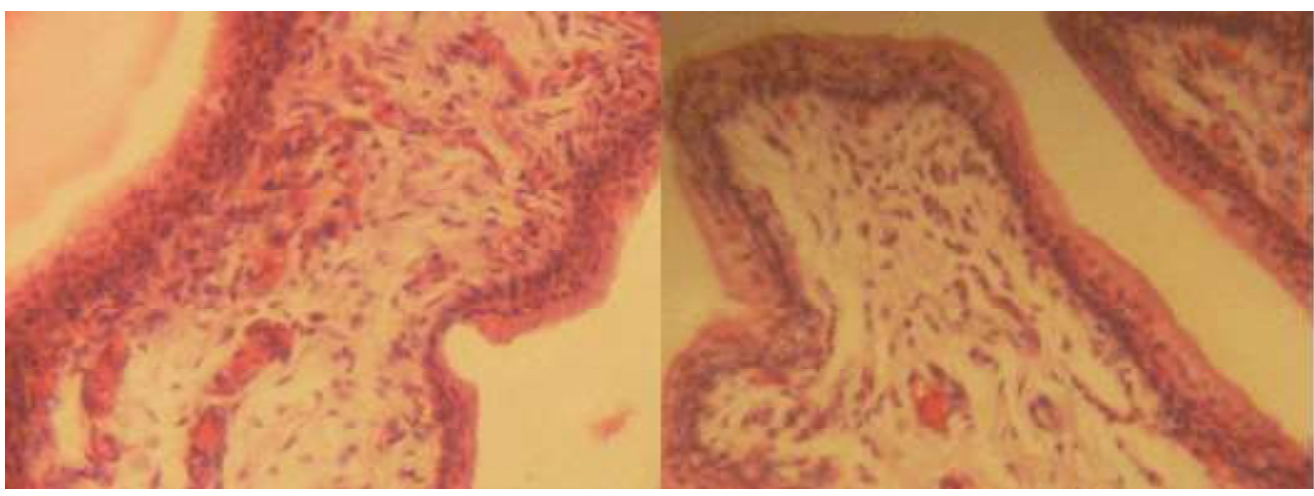

Figura 2. Cortes histológicos de vagina de una alpaca vacía (foto izquierda) y preñada (foto derecha)

Las dimensiones de las células del epitelio vaginal de las alpacas se encuentran en el Cuadro 1. El tamaño de las células fue inferior al reportado en frotices vaginales de gatas (Mills et al., 1979). Asimismo, las células parabasales fueron de menor tamaño que las reportadas para la alpaca $(18-25 \mu \mathrm{m}) \mathrm{e}$ intermedias pequeñas; sin embargo, las células intermedias grandes y superficiales se encontraron dentro el rango descrito por Pedrana et al. (2008). 
Cuadro 1.- Diámetros promedios $(\mu \mathrm{m})$ en células epiteliales en frotis vaginales de alpaca

\begin{tabular}{|c|c|c|}
\hline Tipo de célula & Célula & Núcleo \\
\hline Parabasal & $15.8^{\mathrm{d}}$ & 9.8 \\
\hline Intermedia pequeña & $20.4^{\mathrm{c}}$ & 9.0 \\
\hline Intermedia grande & $33.7^{\mathrm{b}}$ & 11.6 \\
\hline Superficial & $51.4^{\mathrm{a}}$ & \\
\hline
\end{tabular}

Cuadro 2. Índice de maduración de células vaginales en alpacas hembras vacías

\begin{tabular}{lccc}
\hline \multirow{2}{*}{$\begin{array}{l}\text { Tipo de } \\
\text { célula }\end{array}$} & \multicolumn{2}{c}{$\begin{array}{l}\text { Postura ante el } \\
\text { macho }\end{array}$} & \multirow{2}{*}{ Total } \\
\cline { 2 - 3 } & Rechaza & Acepta & \\
\hline Superficiales & $54.2^{\mathrm{a}}$ & $79.5^{\mathrm{a}}$ & 66.9 \\
Intermedias & $29.2^{\mathrm{a}}$ & $15.4^{\mathrm{a}}$ & 22.3 \\
Parabasales & $8.0^{\mathrm{a}}$ & $1.9^{\mathrm{b}}$ & 4.9 \\
Leucocitos & 5.9 & 2.0 & 3.9 \\
Eritrocitos & 2.7 & 1.2 & 2.0 \\
\hline
\end{tabular}

a,b Superíndices diferentes dentro de filas indican diferencia significativa $(p<0.05)$

Cuadro 3. Índice de maduración de células vaginales en alpacas hembras preñadas

\begin{tabular}{lc}
\hline Tipo de célula & Total \\
\hline Superficiales & 12.6 \\
Intermedias & 58.4 \\
Parabasales & 26.9 \\
Leucocitos & 2.1 \\
Eritrocitos & 0 \\
\hline
\end{tabular}

\section{Experimento II}

En hembras vacías, el índice de maduración muestra incremento de células superficiales durante la dominancia estrogénica ( $\mathrm{p}<0.05$; Cuadro 2). El comportamiento de las alpacas hembras frente al macho es atribuido al alto nivel de estrógenos presente en la hembra durante la fase folicular (Bravo, 2002; Quispe et al., 2012). El 66.8\% de células superficiales en las hembras vacías fue similar a lo descrito en pecarís (Mayor et al., 2004), pero inferior a lo reportado en gatos (Mills et al., 1979).

En hembras preñadas, el porcentaje de células superficiales disminuyó mientras el porcentaje de células parabasales e intermedias se incrementó (Cuadro 3), presentando valores similares al anestro en otras especies (Mills et al., 1979; Mayor et al., 2004; Stornelli et al., 2006). Este valor para las células superficiales podría ser utilizado en el diagnóstico de gestación, puesto que es muy diferente a los porcentajes encontrados en alpacas vacías (Cuadro 2).

En el posparto temprano, el epitelio vaginal presenta un alto porcentaje de células intermedias (Cuadro 4), con muy bajos porcentajes de células superficiales, indicando la no existencia de niveles estrogénicos que permitan la maduración de estas células (Mills et al., 1979). Asimismo, se puede observar la alta cantidad de neutrófilos presentes en la vagina al segundo día post parto, debido probablemente a la inflamación del canal de parto (Bravo, 2002), los cuales disminuyen rápidamente a partir del tercer día post parto.

Todas las alpacas presentaron cuerpo lúteo a los 3 y 6 días de la cópula, y a los 9 y 12 días se encontró el embrión además del cuerpo lúteo. Las frecuencias del tipo de célula vaginal se muestran en el Cuadro 5. 
Cuadro 4. Índice de maduración de células vaginales en alpacas hembras durante el posparto temprano

\begin{tabular}{cccccc}
\hline $\begin{array}{c}\text { Días } \\
\text { posparto }\end{array}$ & $\begin{array}{c}\text { Células } \\
\text { superficiales } \\
\%\end{array}$ & $\begin{array}{c}\text { Células } \\
\text { intermedias } \\
\%\end{array}$ & $\begin{array}{c}\text { Células } \\
\text { parabasales } \\
\%\end{array}$ & $\begin{array}{c}\text { Glóbulos } \\
\text { blancos } \\
\%\end{array}$ & $\begin{array}{c}\text { Glóbulos } \\
\text { rojos } \\
\%\end{array}$ \\
\hline 2 & 18.8 & 14.6 & 1.5 & 61.8 & 3.3 \\
3 & 21.0 & 63.7 & 8.0 & 7.0 & 0.3 \\
4 & 21.4 & 67.1 & 6.1 & 5.4 & 0 \\
5 & 27.7 & 67.6 & 4.1 & 0.6 & 0 \\
\hline
\end{tabular}

Cuadro 5. Índice de maduración de células vaginales a los 3, 6, 9 y 12 días pos-cópula en alpacas hembras con embrión en el tracto reproductivo

\begin{tabular}{lcccc}
\hline Tipos celulares & Día 3 & Día 6 & Día 9 & Día 12 \\
\hline Superficiales & 65.3 & 56.9 & 42.4 & 28.0 \\
Intermedias & 32.8 & 37.6 & 52.2 & 59.5 \\
Parabasales & 1.9 & 5.5 & 5.4 & 12.5 \\
\hline Total (\%) & 100 & 100 & 100 & 100 \\
\hline
\end{tabular}

La alta frecuencia de células superficiales en día 3 pos-cópula ante la presencia de un cuerpo lúteo en desarrollo (Bravo, 2002) podría estar reflejando una descamación acelerada ante la elevada concentración de estrógenos en los días previos. Este tipo de célula comienza a descender gradualmente de forma tal que el día 12 pos-cópula se observa una dominancia de las células intermedias, lo cual es compatible con un estado de gestación. Las células parabasales, por otro lado, presentaron una baja frecuencia durante este periodo.

\section{Experimento III}

Los cortes histológicos de vagina en una alpaca vacía (Figura 2) muestran una mucosa cubierta con epitelio plano estratificado homogéneo con apariencia pavimentosa en la mayor parte de su extensión (Feldman y Nelson, 2007). Se llega a observar hasta sie- te y más capas de células y zonas con descamación celular, posiblemente por influencia estrogénica ante la presencia de folículos desarrollados (Diehl y Day, 1973; Jones y Edgren, 1973); además de la presencia de pliegues, a veces pronunciados, a manera de criptas. La submucosa se encuentra organizada en fibras regulares y de manera laxa con bastante irrigación y con presencia de acumulaciones linfocíticas (Gartner y Hiatt, 2002; Rodríguez et al., 2004; Xelhuantzi et al., 2004), en tanto que la capa muscular se encuentra organizada en dos capas delgadas y cubiertas por células mesoteliales (Gartner y Hiatt, 2002).

En el caso de las alpacas preñadas (Figura 2), se observa una mucosa con epitelio estratificado plano (Feldman y Nelson, 2007), con cinco o menos capas celulares, indicando la ausencia de estrógenos capaces de producir proliferación celular (Diehl y Day, 1973; 
Jones y Edgren, 1973); sin embargo, en algunas zonas se observa la apariencia de un epitelio seudoestratificado con células cilíndricas y zonas engrosadas con células cúbicas hipertrofiadas, lo cual sería un comportamiento celular sui generis en esta especie. El epitelio se observa delgado, con numerosas criptas o pliegues a manera de rugosidades (Rodríguez et al., 2004; Xelhuantzi et al., 2004), con células grandes y globosas y células caliciformes. La submucosa presenta tejido conectivo poco vascularizado, delgado y con contenido linfocitario. La capa muscular se encuentra aumentada en grosor y la disposición de las fibras se observa desordena$\mathrm{da}$, posiblemente debido a que ambos animales fueron multíparos (Gartner y Hiatt, 2002; Rodríguez et al., 2004).

\section{Conclusiones}

- En los frotis vaginales de alpacas en las diversas fases del ciclo reproductivo se observan células similares a las reportadas en otras especies, por lo que dichos protocolos de tinción y evaluación podrían utilizarse en evaluaciones de frotis vaginales de alpacas.

- Los datos obtenidos a partir de frotis vaginales en alpacas indican el estadio del epitelio vaginal de acuerdo al estado reproductivo del animal, habiendo marcadas diferencias entre alpacas vacías y alpacas preñadas.

- El frotis vaginal podría ser utilizado como un método complementario de diagnóstico de gestación, al ser una técnica no invasiva.

\section{Literatura Citada}

\section{Bekyürek T, Liman N, Bayram G.} 2002. Diagnosis of cycle by means of vaginal smears method in the chinchilla (Chinchilla lanigera). Lab Anim 4: 5160. doi: 10.1258/0023677021911768
2. Bravo W. 2002. The process reproductive of South American camelids. Salt Lake City, USA: Seagull Printing. 100 p.

3. Dellman H, Brown E. 1980. Histología veterinaria. $4^{\mathrm{a}}$ ed. España; Acribia. 529 p.

4. Diehl JR, Day BN. 1973. Utilization of frozen sections with the vaginal biopsy technique for early pregnancy diagnosis in swine. J Anim Sci 37: 114-117.

5. Feldman EC, Nelson RW. 2007. Endocrinología y reproducción en perros y gatos. 2a ed. México: McGraw-Hill Interamericana. $1218 \mathrm{p}$.

6. Gartner L, Hiatt J. 2002. Texto atlas de histología. $2^{a}$ ed. México: McGrawHill Interamericana. $538 \mathrm{p}$.

7. Hawkins LE, Darlow AE. 1934. Hystology of the reproductive tract in the ewe during the oestrous cycle. J Anim Sci 1: 274-277. doi: 10.2527/jas1934.$19341274 \mathrm{x}$

8. Jones RC, Edgren RA. 1973. The effects of various steroids on the vaginal histology in the rat. Fertil Steril 24: 284291.

9. Mayor P, Gálvez H, Guimarães DA, Lopez-Bejar M. 2004. Características del estro de la hembra de pecarí (Tayassu tajacu) del este amazónico. Memorias VI Congreso Internacional sobre Manejo de Fauna Silvestre en Amazonia y Latinoamérica. Iquitos, Perú.

10. Mills JN, Valli VE, Lumsden JH. 1979. Cyclical changes of vaginal cytology in the cat. Can Vet J 20: 95-101.

11. Mitchell S, Jones SM. 1975. Diagnosis of pregnancy in marmosets (Callitrix jachus). Lab Anim 9: 46-56.

12. Pedrana G, Sena $X$, Cuñarro B, Vitarella F, Fumagalli F, Lima M, Mernies B. 2008. Caracterización morfológica de la citología vaginal exfoliativa en ungulados del orden Artiodactyla. Resúmenes IX Simposio iberoamericano sobre conservación y utilización de recursos zoogeneticos. Mar del Plata. Argentina. 
13. Piñeiro C, Gorraiz JM, Martin N, Amigo JM. 1995. Green book del veterinario. $2^{\mathrm{a}}$ ed. Madrid, España: Ed. Marban. $130 \mathrm{p}$.

14. Porto RR, Vasconcelos T, Ferreira F, Moreira J, Torres de Souza J. 2007. Perfil citologico vaginal de ovelhas da raca Santa Inés no acompanhamento do ciclo estral. Cienc Anim Bras 8: 521-527.

15. Quispe A, Coila P, Pacheco JI, Catacora N, Mamani R, Ruelas D. 2012. Niveles de estradiol en líquido folicular en alpacas según tamaño y su relación con niveles sanguíneos. Resúmenes VI Congreso Mundial de Camélidos Sudamericanos. Chile.

16. Ríos P, Echevarría L, Santillán G, Gálvez H. 1999. Imagen citológica vaginal durante el ciclo ovárico en monos nocturnos Aotus nancymae y A. vociferans. Rev Inv Vet Perú 10(2): 1721. doi: 10.15381/rivep.v10i2.6695

17. Rodríguez J, García M, Fajardo V, Martínez M. 2004. Histología del tracto urogenital inferior de la coneja doméstica: relación con la edad y número de par- tos. Resúmenes XXVIII Congreso Nacional de Histología. México DF.

18. Stornelli MA, Savignone CA, Tittarelli MC, Stornelli MC. 2006. Citología vaginal en caninos: metodología y aplicaciones clínicas. Vet Cuyana 1(1): 15-21.

19. Thrall MA, Olson PN. 2003. Sistema reproductivo. En: Cowell R, Tyler RD, Meinkoth J (eds). Citología y hematología diagnostica en el perro y el gato. $2^{\mathrm{a}}$ ed. Barcelona, España: Ed Mosby. p 318350 .

20. Williams E, Thorne T, Kwiatkowski D, Lutz K, Anderson S. 1990. Comparative vaginal cytology of the estrous cycle of black-footed ferrets (Mustela nigripes), Siberian polecats (Mustela eversmanni) band domestic ferrets (Mustela putorius furo) $\mathrm{J}$ Vet Diagn Invest 4: 38-44. doi: 10.1177/ 104063879200400109

21. Xehuantzi N, Rodríguez J, Pacheco P, Martínez M. 2004. Estudio histológico de la vagina pélvica de la coneja domestica. Resúmenes XXVIII Congreso Nacional de Histología. México DF. 